\title{
Progress in the Discovery of Small Molecule Modulators of DeSUMOylation
}

\author{
Shiyao Chen, Duoling Dong, Weixiang Xin and Huchen Zhou*
}

School of Pharmacy, Shanghai Jiao Tong University, Shanghai, China.

*Correspondence: hczhou@sjtu.edu.cn

https://doi.org/10.21775/cimb.035.017

\begin{abstract}
SUMOylation and DeSUMOylation are reversible protein post-translational modification (PTM) processes involving small ubiquitin-like modifier (SUMO) proteins. These processes have indispensable roles in various cellular processes, such as subcellular localization, gene transcription, and DNA replication and repair. Over the past decade, increasing attention has been given to SUMOrelated pathways as potential therapeutic targets. The Sentrin/SUMO-specific protease (SENP), which is responsible for deSUMOylation, has been proposed as a potential therapeutic target in the treatment of cancers and cardiac disorders. Unfortunately, no SENP inhibitor has yet reached clinical trials. In this review, we focus on advances in the development of SENP inhibitors in the past decade.
\end{abstract}

\footnotetext{
Introduction

Post-translational modification (PTM) of proteins is a crucial process for the regulation of biological growth and the stress response, and operates via extremely sophisticated mechanisms. There are at least 20 types of PTM in eukaryotes, such as ubiquitination, phosphorylation, methylation, glycosylation, and acetylation. Among them, a reversible modification process involving small ubiquitin-like modifier (SUMO) proteins, which is thus termed SUMOylation, has an indispensable role in various cellular processes, such as modulation of protein stability, subcellular localization,
}

protein-protein interactions, gene transcription, genome integrity, and DNA replication and repair (Wilkinson and Henley, 2010; Vierstra, 2012; Bailey et al., 2016). In 1995, Meluh and Koshland (1995) identified Smt3 in Saccharomyces cerevisiae, which is the earliest report within this filed. Two years later, based on the sequence similarity between ubiquitin and a new $11.5-\mathrm{kDa}$ protein, ubiquitin/SMT3, the name SUMO was formally proposed for the first time (Mahajan et al., 1997).

Although SUMO modification is closely related to the progression of various diseases, such as cancers and cardiac disorders, it has aroused increasing attention as a potential therapeutic target in recent years, especially concerning the Sentrin/ SUMO-specific protease (SENP), which is the key regulator of deSUMOylation. Unfortunately, no SENP inhibitor has yet reached clinical trials. In this review, we focus on advances in the development of SENP inhibitors within the past decade. The opportunities and challenges are discussed.

\section{SUMO modification and its cellular functions}

\section{SUMO modification cycle}

SUMO is a class of small proteins that are highly conserved during evolution. Distinct from yeast and invertebrates, which have only a single SUMOencoding gene, there are at least four SUMO isoforms in vertebrate genomes, SUMO1-4. SUMO2 and SUMO3 are commonly referred to as 
SUMO2/3 because of their high sequence similarity (97\%), whereas SUMO1 is less closely related to SUMO2/3 (almost 50\%), requiring different activating and conjugating enzymes (Kerscher et al., 2006). Both SUMO1 and SUMO2/3 show certain preference for their substrates. For instance, ran GTPase activating protein 1 (RanGAP1) is a typical substrate of SUMO1, while Topoisomerase II is predominantly modified by SUMO2/3. Some other proteins, such as promyelocytic leukaemia (PML) seem to be insensitive to SUMO isoforms. Besides, their ability to form SUMO chains is also diverse. However, whether SUMO4 is capable of protein processing or conjugation remains unclear (Guo et al., 2004). In eukaryotic cells, all SUMOs are translated as immature precursors that must be transformed to the mature state before SUMOylation, which is initiated by SENPs through their SUMO peptidase activity (Park et al., 2011). Under the action of certain SENPs, about 10 amino acids at the C-terminus of SUMO precursors are removed and thus the crucial diglycine (GlyGly) binding site motif in SUMOs is exposed for the later conjugation (Johnson, 2004) (Fig. 4.1).

Similar to ubiquitination, SUMO modification of substrate proteins also requires a series of enzymatic reactions and results in the formation of an isopeptide bond between the SUMO C-terminal carboxyl group and the $\varepsilon$-amino group of a lysine residue in the substrate. The first step of SUMO modification is catalysed by SUMO activating enzyme E1. In human cells, SUMO E1 is a heterodimer composed of two subunits, SUMO1 activating enzyme subunit 1 (SAE) 1 and SAE2. The former can be further decomposed into SAE1a and SAE1b (Johnson, 2004). During this step, an ATP molecule is hydrolysed for energy supplementation and an E1 SUMO high-energy thioester bond is formed between the glycine carboxyl group (SUMO C-terminal) and the sulfhydryl group (SAE2 cysteine) (Park et al., 2011). Subsequently, the activated SUMO is transferred to a cysteine residue in the SUMO conjugating enzyme E2 to form a new thioester bond E2 SUMO. It is worth noting that until now, only one SUMO E2, named ubiquitin conjugating enzyme E2 I (UBC9), has been identified, which is in sharp contrast with the tens of $\mathrm{E} 2$ enzymes that act in unique combinations. Although UBC9 can directly react with a certain portion of the substrates and transfer SUMO to the lysine residue in the target protein, thereby completing the SUMOylation process in absence of SUMO E3 ligase (Park et al., 2011), it has been proven that E3 ligase can enhance SUMO conjugation in two ways (Miura and Hasegawa, 2010). On the one hand, via interaction with the substrate, SUMO E3 ligase recruits the E2 SUMO thioester and the substrate into a complex, which narrows
A)

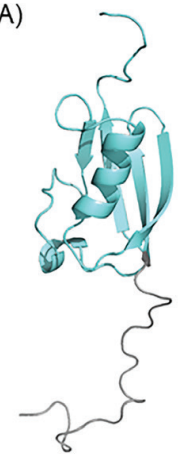

D)

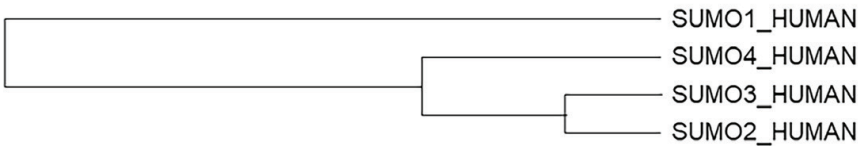

C)
B)

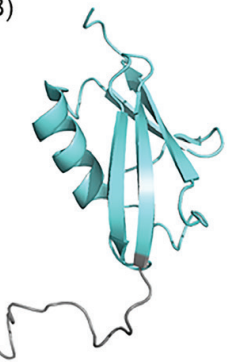

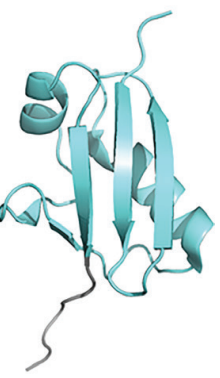

Figure 4.1 The crystal structure of SUMOs and their evolutionary relationships. (A) SUMO1 (PDB: 1A5R). (B) SUMO2 (PDB: 2N1W) (C) SUMO3 (PDB: 1U4A). The flexible N-terminal extension is coloured grey. (D) Evolutionary relationship of SUMO family members. 
their distance and contributes to the specificity of transfer. On the other hand, the catalytic activity of E2 is up-regulated in the presence of E3 ligase, which contributes to the efficiency of SUMOylation. There are four E3 ligases in yeast, namely Siz1, Siz2, Mms21, and Zip3; whereas mammals possess ten, including three protein inhibitor of activated STAT (PIAS) family members, Methyl methanesulfonate sensitivity gene 21 (MMS21), RAN binding protein 2 (RanBP2), Pc2 (also known as Keratin 17) and OP1 binding arginine/serine rich protein (TOPORS).

As mentioned above, the SUMO modification is a reversible process. To complete the SUMO modification cycle, deconjugation of SUMO from SUMOylated protein substrates is also indispensable and is catalysed by the SENP family. Apart from its function as a maturation enzyme, SENP is also capable of the cleaving the isopeptide bond formed between the C terminus of SUMO and the $\varepsilon$-amino group of the lysine residue in the target, thereby promoting the release of SUMO (Mukhopadhyay and Dasso, 2007). The members of SENPs were first discovered in Saccharomyces cerevisiae and named as Ulp1 and Ulp2, while the human SENP family was characterized later, including SENP1, SENP2, SENP3, SENP5, SENP6, and SENP7 (Li and Hochstrasser, 1999, 2000; Hickey et al., 2012). Interestingly, although the processing and deconjugation of SUMO is achieved by the same group of proteases, some SENPs act only as deSUMO enzymes and do not participate in
SUMO maturation (Saracco et al., 2007; Castro et al., 2016) (Fig. 4.2).

\section{SUMO interaction domains}

The recognition and conjugation of most SUMOylated substrates by SUMO E2 is achieved via a short consensus modification motif $\psi \mathrm{Kx}(\mathrm{D} / \mathrm{E})$ characterized in multiple target proteins, in which $\psi$ represents a large hydrophobic residue, whereas $\mathrm{x}$ can be any amino acid (Rodriguez et al., 2001). Acting directly on UBC9, these residues have a crucial impact on the stability of the interaction between SUMO E2 and the target. In the crystal structure of a SUMO consensus motifs-containing protein-UBC9 complex, the modification motifs adopt an extended conformation, which limits the acceptor lysine in the UBC9 hydrophobic groove. Electrostatic interactions as well as hydrogen bonding between UBC9 and the amino acids adjacent to the acceptor lysine also contribute to its recognition (Lin et al., 2002). In addition to the four-amino acidlength canonical consensus motifs, several variants have been identified, such as motifs with additional elements nearby. For instance, phosphorylation of certain sites, termed as phosphorylation-dependent SUMO motifs (PDSMs), promotes up-regulation of SUMOylation both in vitro and in vivo, and was first discovered in heat shock proteins. There are also negatively charged amino acid dependent SUMO motifs (NDSMs), which possess negatively charged residues. Reports of novel SUMO consensus motifs, such as inverted consensus motifs and

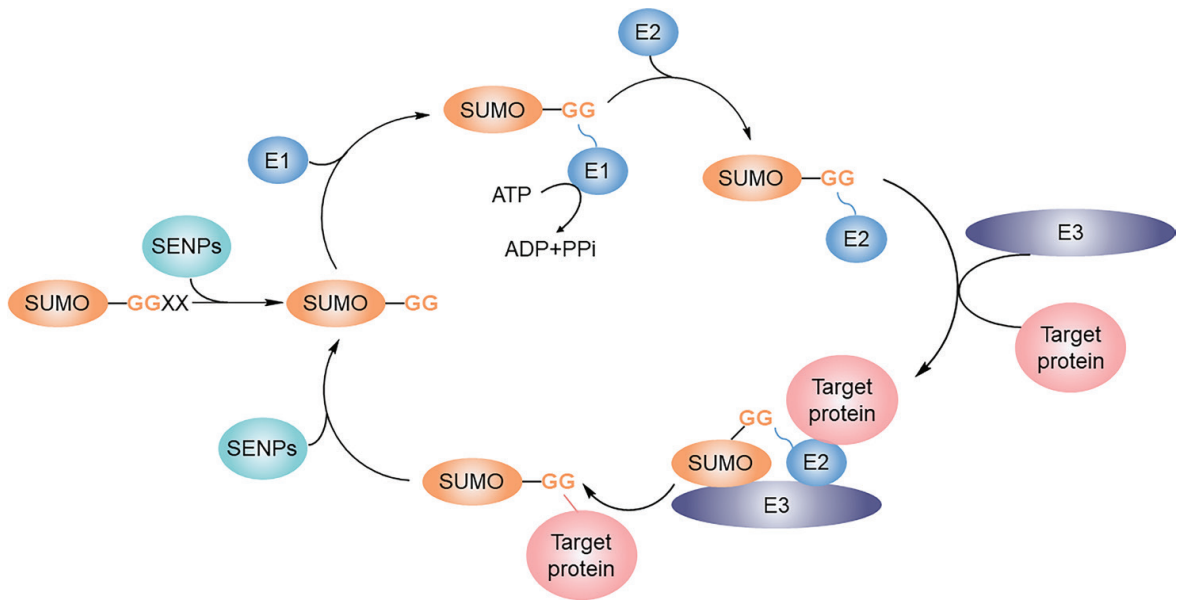

Figure 4.2 The SUMOylation and deSUMOylation cycle. 
motifs combined with an $\mathrm{N}$-terminal hydrophobic cluster with distinct polarity, emphasizes the importance of detailed research into the diversity of SUMO conjugation sites (Hietakangas et al., 2003; Mohideen et al., 2009; Matic et al., 2010). Furthermore, such consensus motifs do not strictly follow the sequence order or geometrical requirements, for example, the lysine in the $\alpha$-helix of ubiquitin conjugating enzyme E2 K (E2-25K) (Pichler et al., 2005; Knipscheer et al., 2008).

Another SUMO-binding domain that has been studied in depth is the SUMO-interacting motif (SIM), which mediates non-covalent interactions between SUMO and proteins containing SIMs (Merrill et al., 2010). It is typically composed of a hydrophobic core with four consensus hydrophobic residues, $\mathrm{V} / \mathrm{I}-\mathrm{x}-\mathrm{V} / \mathrm{I}-\mathrm{V} / \mathrm{I}$ or $\mathrm{V} / \mathrm{I}-\mathrm{V} / \mathrm{I}-\mathrm{x}-\mathrm{V} / \mathrm{I} / \mathrm{L}$, where $\mathrm{x}$ can be any amino acid, flanked by acidic residues providing the necessary polarity (Merrill et al., 2010). When complexed with SUMO, SIMs adopt either a parallel or antiparallel $\beta$-conformation to the SUMO $\beta$-sheet, exposing the hydrophobic side chains to the hydrophobic pocket on the SUMO surface. Molecular dynamic simulations demonstrated higher stability of the complex with the antiparallel orientation together with better tolerance to sequence changes, possibly because of the establishment of more backbone-mediated interactions (Conti et al., 2014; Jardin et al., 2015). In addition, a subclass of SIMs possessing serine residues as phosphorylation sites, adjacent to the hydrophobic core, has been characterized in PML, exosome component 9 (EXO9), and PIAS proteins (Stehmeier and Muller, 2009). Phosphorylated by casein kinase 2 (CK2), these phospho-SIMs enhance their non-covalent binding to SUMO through electrostatic interactions between the negatively charged phosphorylated serine and positively charged lysine on the SUMO surface, which is also presumed to further affect the specificity of different SUMO isoforms.

SIMs are found in many proteins, including SUMO substrates and binding partners, SUMOtargeted ubiquitin ligases (like Slx8-rING finger protein $(\mathrm{rfp})$ in $S$. pombe and Slx5-Slx8 in S. cerevisiae), as well as all known SUMO E3s, serving as a crucial regulator in various cellular processes. Although found in ubiquitin-specific protease 25 (USP25), a substrate for SUMOylation, in which the SIMs were observed to contribute to a modification preference for SUMO2/3, their structural determinants for such specificity remain to be verified and the measured affinity between SIMs and different SUMO isoforms did not appear to be significantly different (Meulmeester et al., 2008; Sekiyama et al., 2008; Kung et al., 2014). Human ZNF451, a SUMO E3 ligase, contains two SIMs separated by a Pro-Leu-Arg-Pro sequence in its catalytic region, which provides support for the effect of SIMs on E3 ligase activity (Cappadocia et al., 2015). The N-terminal SIM of ZNF451 maintains the donor SUMO in a closed conformation, whereas its C-terminal SIM combines with a second SUMO on the reverse side of UBC9, which ensures direct contact between certain residues and UBC9. ZNF451 itself is also a target of SUMO modification. SUMOylation occurs close to the catalytic module, which causes an increase in its activity as a SUMO E3 ligase (Hendriks and Vertegaal, 2016). Furthermore, it was proposed recently that the SUMO modification targets entire groups of interacting proteins rather than individual proteins, thus the presence of SIMs in the substrates has a key role in protein-group SUMOylation via multiple SUMO-SIM interactions (Jentsch and Psakhye, 2013).

Other types of SUMO interaction domains have also been reported, for instance the $\mathrm{ZZ}$ zinc finger domain that interacts with SUMO in a zinc-dependent manner and the zinc-independent MYM-type zinc finger domain (Danielsen et al., 2012; Guzzo et al., 2014). However, the latter seems to bind to the same site in SUMO as SIMs, such that the destruction of the SUMO-SIM interaction simultaneously affects the stability of the SUMO-MYM interaction (Guzzo et al., 2014).

\section{Cellular roles of SUMOylation}

To date, numerous studies on the essential role of SUMOylation in normal cell homeostasis have been carried out, the majority of which were based on the regulation of transcription. Early studies demonstrated that SUMOylation is closely related to transcriptional repression (Gill, 2005). One of the hypotheses attributes this to the recruitment of transcriptional corepressors, such as the histone deacetylase 1 and 2 (HDAC) $1 / 2$ complex (Yang and Sharrocks, 2004). SUMOylation might also be involved in the regulation of factors related to RNA polymerase Pol II, an emerging 
finding proposed more recently (Neyret-Kahn et al., 2013; Niskanen et al., 2015). As reported by Yu et al. (2018), SUMO and MYC have opposite effects on global transcription by controlling the level of SUMO modification of cyclin dependent kinase 9 (CDK9), which is the catalytic subunit of positive transcription elongation factor $b$ (P-TEFb) complex (Yu et al., 2018). SUMOylation of CDK9 interrupts its binding to the regulatory subunit Cyclin T1, thereby causing a pause in the formation of the active $\mathrm{P}-\mathrm{TEFb}$ complex, which ultimately blocks global gene expression. By contrast MYC antagonizes SUMOylation processing in combination with PIAS in a CDK9-competitive way, serving as a broad-spectrum promoter for cellular transcription.

SUMOylation is also implicated in the modulation of cellular stress responses, like the endoplasmic reticulum (ER) stress response, viral infections, nutrient response, and especially, the DNA damage response (Enserink, 2015). Ubiquitin-SUMO crosstalk occurs extensively in signalling responses to double-strand breaks (DSBs). One example is ring finger protein 4 (RNF4), a SUMO targeting ubiquitin ligase (STUbLs) in mammalian cells. Containing tandem SIMs in its N-terminus, RNF4 is capable of recognizing poly-SUMOylated proteins and promoting K48-linked ubiquitination (Galanty et al., 2012). In addition, RNF4 also catalyses ubiquitin conjugating enzyme E2N (UBC13)dependent K63-linked polyubiquitination (Yin et al., 2012). Phenotypically, cells lacking RNF4 show defective RAD51 recombinase (RAD51) loading, leading to blockade of chromosome homologous recombination, for which inefficient exchange of replication protein A (RPA) with RAD51 is caused by the decrease of SUMO-modified RPA turnover from single-stranded DNA (ssDNA) may be an acceptable explanation (Galanty et al., 2012; Yin et al., 2012).

\section{DeSUMOylation and SENPs}

As stated above, the members of the SENP family have a dual effect as maturation enzymes in precursor processing and SUMO deconjugases. In the body, SUMOylation and deSUMOylation are require for a dynamic equilibrium relationship, while the balance between SUMO and deSUMO modification of proteins in diverse cellular compartments is mainly attributed to SENPs. Based on their sequence homology, substrate specificity, and subcellular localization, the six SENP isoforms in mammals, SENP1, SENP2, SENP3, SENP5, SENP6 and SENP7, can be classified into three sub-families: SENP1 and SENP2, SENP3 and SENP5, and SENP6 and SENP7 (Gong and Yeh, 2006). In terms of their evolutionary relationship, the first two families can also be classified as the Ulp1 branch, while SENP6 and SENP7 belong to the Ulp2 branch (Mukhopadhyay and Dasso, 2007).

\section{Structural characteristics}

Compared with the variable $\mathrm{N}$-terminus, which contributes to the differences in spatial distribution and substrate specificity among distinct SENP isoforms, the $\mathrm{C}$-terminal regions among all six SENPs seem to be more conserved, containing a cysteine protease catalytic domain that is approximately 250 amino acids in length (Hickey et al., 2012). Currently, only the crystal structure of the catalytic domain of SENP1 (2IYC, 2IY1, 2IYD, 2IY0) coupled with SENP2 (1TH0, 1TGZ, 2IO0, 2IO1, 2IO2, 2IO3), and SENP7 (3EAY) has been reported, either in apo form or in complex. Composed of the typical catalytic triad (Cys-HisAsp) (Cys603, His533 and Asp550 for SENP1; Cys-548, His-478, and Asp-495 for SENP2; His794, Cys-926 and Asp-873 for SENP7), which is analogous to other cysteine proteases, the crystal structures of these three SENPs are quite similar (Kumar and Zhang, 2015). The structures highlight that this catalytic domain is indispensable for the hydrolytic activity in precursor processing and deSUMOylation, such that the replacement of the active-site cysteine residue with serine in human SENP1 damaged to its function (Xu et al., 2006). Meanwhile, a narrow tunnel lined by Trp residues in SENP1 is critical for the positioning of the di-Gly motif, while the closing it contributes to the orientation of the sessile bond, thus forming an unstable kink in the linkage to the SUMO substrate proteins, which is believed to promote cleavage (Shen et al., 2006). Among the catalytic triads, cysteine, as the main catalytically active site, is the most commonly used target for the development of SENP inhibitors (Fig. 4.3).

In particular, the structure of the catalytic domain of SENP7, consisting of amino acid 

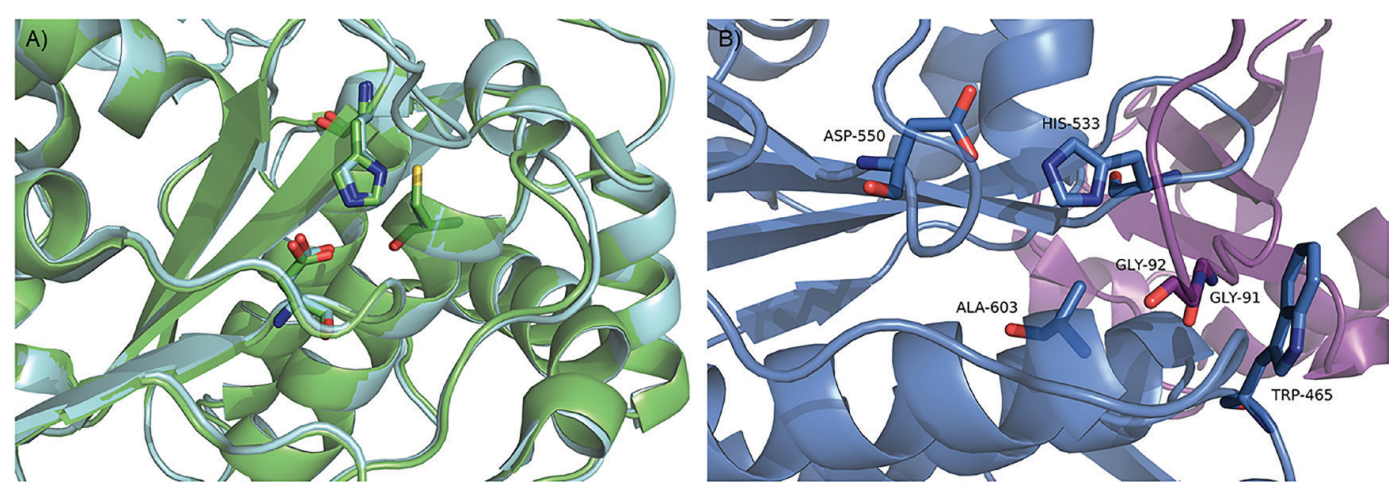

Figure 4.3 Structures of SENPS. (A) Details of the SENP1 catalytic triad. (B) Details of the SENP1 catalytic triad and the Trp tunnel in the SENP1-SUMO1 complex. SENP1 is shown in blue and SUMO1 is shown in in purple (PDB: 2IY1).

residues 662-984, reveals its relationship between the SENP/Ulp protease family and other Cys-48 cysteine proteases. Consistent with its substrate specificity, SENP7 has a unique catalytic structure that is apparently different from SENP1 and SENP2, including the absence of the N-terminal $\alpha$-helix, the insertion of four conserved loops, and the extension of several secondary structure elements (Lima and Reverter, 2008). Loop1 is highly conserved in SENP6 and SENP7, suggesting its potential contribution to catalytic activity, which was subsequently proved (Lima and Reverter, 2008). Compared with wild-type SENP7, mutants lacking loop1 show an apparent defect in enzyme activity of SENP7 for both precursor processing and SUMO-deconjugation. Besides, Alegre and Reverter (2011) identified that the position of Asp71, coupled with Asn68 of SUMO2, is close to loop1 of SENP7 in the crystal structure of their complex, indicating possible polar interactions. Further studies showed that these two key amino acids are directly responsible for the preference of SENP7 for SUMO2/3 through interaction with loop1.

\section{Substrate specificity}

\section{SENP1 and SENP2}

SENP1 and SENP2 show broad specificity for SUMO1/2/3. SENP2 has a similar activity to SENP1 when overexpressed; however, it prefers SUMO2 over SUMO1 for deconjugation and has a relatively poor effect on SUMO3. The mechanisms of action of these two isoforms are distinct, for example, although both SENP1 and SENP2 can regulate c-Jun-dependent transcription, SENP1 works by deSUMOylation of p300 while SENP2 targets PML (Best et al., 2002; Cheng et al., 2005). Emerging research suggests that SENP1 regulates the stability of hypoxia inducing factor 1 (HIF1), while SENP2 does not, clearly indicating that both of them have their own specific substrates (Yeh, 2009).

\section{SENP3 and SENP5}

SENP3 and SENP5 share high sequence identity and the same localization; it is reasonable to deduce that they may have similar substrate selectivity (Gong and Yeh, 2006). Compared with SUMO1, SENP3 and SENP5 show a prominent preference for SUMO2/3. The stability of SENP3 is regulated by CHIP, which is the carboxyl-terminus of heat shock protein family A (HSP70) member 8 (HSC70)-interacting protein through the heat shock protein 90 (HSP90)-independent ubiquitinproteasome pathway (Yan et al., 2010). However, in response to mild oxidative stress, SENP3 undergoes thiol modification, by which HSP90 is recruited, and subsequently its degradation is repressed. In liver cancer, SENP3 accumulates and accelerates disease progression by responding to the abnormal redox background (Yan et al., 2010). SENP5 is crucial in cell mitosis and/or cytokinesis, and the absence of SENP5 causes proliferation inhibition and abnormal nuclear morphology (Di Bacco et al., 2006). 


\section{SENP6 and SENP7}

Among the six human SENP isoforms, the catalytic domains of SENP6 and SENP7 are the most diverse, especially the insertion of loop 1 , which mediates the specific interaction with SUMO2/3conjugated substrates. As a consequence, SENP6 and SENP7 preferentially act on SUMO2/3, and moreover, are more effective in cleaving diSUMO2/3 or poly-SUMO2/3 chains attached to lysine residues (Alegre and Reverter, 2011; Lima and Reverter, 2008).

SENPs fail to achieve the proteolytic processing of SUMO4 precursor molecule in vivo, which is indispensable for its maturation; therefore, the posttranslational modification of substrate proteins by SUMO4 has not yet been observed (Owerbach et al., 2005). In precursor SUMO4, Pro-90 replaces Gln in SUMO1-3, which causes a conformational restriction that might keep the peptide bond to be cleaved distal from the catalytic site of SENP and thus disrupt the maturation process (Békés et al., 2011). A P50Q single amino acid mutant of precursor SUMO4 made it amenable to SENP2 cleavage, as did another mutant, G63D (Liu et al., 2014). Besides, although all six SENP isoforms possess SUMO deconjugation/isopeptidase activity, only SENP1, SENP2, and SENP5 can carry out SUMO maturation.

\section{Cellular localization}

Different SUMO isopeptidases have characteristic subcellular distributions, which is closely related to the varied lengths and specificities of the N-terminal domains, which seems to contribute to substrate specificity. SENP1 consists of 644 amino acids, with a nuclear localization signal (NLS) and nuclear export-signal (NES) at its N-terminus and C-terminus, respectively (Bailey and O'Hare, 2004). The structure of SENP2 is quite similar. Interacting with components of the nuclear pore complex, SENP1 and SENP2, coupled with Ulp enzyme, which was identified in yeast, gather on the nuclear envelope and accumulate in distinct subnuclear structures (Goeres et al., 2011). Although they are excluded from the nucleolus, substantial amounts of SENP1 and SENP2 are observed in nuclear foci that partially overlap with PML bodies. During mitosis, SENP1 and SENP2 are redistributed from the nuclear envelope to the kinetochore (CubeñasPotts et al., 2013). Notably, measuring a series of its mutants with interspecies heterokaryons indicated that SENP2 shuttles between the nucleus and the nucleoplasm, which can be inhibited by mutation of its NES or treatment with leptomycin B (LMB), in spite of its predominantly nuclear localization (Itahana et al., 2006). In addition, diverse splice variants of SENP2 show specific subcellular localizations (Hickey et al., 2012).

Both SENP3 and SENP5 are compartmentalized in the nucleolus, the function of which is to act on proteins involved in the early stage of ribosome maturation (Haindl et al., 2008; Yun et al., 2008). SENP3, also known as SMT3IP1 or SMTB1 in mice, comprises 574 and 568 amino acids, respectively. SENP3 contains unique sequences in its $\mathrm{N}$-terminus, including glutamate clusters (residues 74-86) and two regions rich in arginine (residues 119-122, 147 and 153), which may account for its nucleolar localization (Nishida et al., 2000). SENP5 comprises 755 amino acids with an extended sequence at its $\mathrm{N}$-terminus, a truncated variant of which co-localizes with the PML (Di Bacco et al., 2006). In addition, subfractions of SENP3 and SENP5 are also found in the nucleoplasm and cytoplasm. In particular, SENP5 translocates to the surface of mitochondria before the rupture of the nuclear envelope during G2/M stage of the cell cycle (Zunino et al., 2009). By contrast, SENP6 and SENP7 are generally concentrated in the nucleoplasm (Table 4.1).

\section{Cellular pathways controlled by SENPS}

\section{Cell cycle}

Considering the spatiality and temporality of SUMOylation in mitosis, it is easy to associate SENPs with cell cycle progression. In budding yeast, Ulp1, which acts on Smt3 and SUMO1-conjugated proteins, exhibits an essential role in the transition from G2 to M phase (Li and Hochstrasser, 1999). Knockdown of SENP1 causes the failure of sister chromatid separation and arrests progression at M phase; however, overexpression of SENP2 also decreases global SUMOylation, which leads to prometaphase arrest because of defects in targeting the microtubule motor protein centromere protein E (CENP-E) to kinetochores (Zhang et al., 2008; Cubeñas-Potts et al., 2013). Moreover, Mukhopadhyay and Dasso (2010) identified SENP6 as a key 
Table 4.1 Properties of SENP isoforms

\begin{tabular}{|c|c|c|c|}
\hline $\begin{array}{l}\text { SENP } \\
\text { isoform }\end{array}$ & $\begin{array}{l}\text { Substrate } \\
\text { preference }\end{array}$ & Cellular localization & Function \\
\hline SENP1 & SUMO1/2/3 & Nuclear pore and nuclear foci & Deconjugation/isopeptidase; precursor processing \\
\hline SENP2 & SUMO2/3 > SUMO1 & $\begin{array}{l}\text { Nuclear pore and nuclear foci; } \\
\text { cytoplasm }\end{array}$ & Deconjugation/isopeptidase; precursor processing \\
\hline SENP3 & SUMO2/3 & Nucleolus & Deconjugation/isopeptidase \\
\hline SENP5 & SUMO2/3 & Nucleolus & Deconjugation/isopeptidase; precursor processing \\
\hline SENP6 & SUMO2/3 & Nucleoplasm & Deconjugation/isopeptidase; chain-editing \\
\hline SENP7 & SUMO2/3 & Nucleoplasm & Deconjugation/isopeptidase; chain-editing \\
\hline
\end{tabular}

regulator of inner kinetochore assembly. Deletion of SENP6 directly led to the mis-localization of inner kinetochore proteins (IKPs) in Hela cells, which caused chromosome misalignment with missegregation, and subsequently delayed cell cycle progression. By antagonizing the STUbL pathway, SENP6 functions as a protector of IKP, keeping it away from $S$ phase degradation (Mukhopadhyay and Dasso, 2010). The mitotic substrate specificity of SENPs remains to be determined.

\section{Transcription}

Among the known substrate proteins of SUMOylation, nuclear proteins occupy a considerable proportion, which participate in the transcriptional regulation of genes and chromatin dynamics. In most cases, the conjugation of core histones is associated with transcriptional silencing, and SUMOylated transcription factors or transcriptional co-regulators are thought to induce a decrease or even inhibition of gene activation (Wotton et al., 2017). Correspondingly, deSUMOylation, mediated by SENPs, facilitates transcription (Niskanen and Palvimo, 2017; Wotton et al., 2017). For instance, SENP3 affects the assembly of the MLL1/2 (also known as lysine methyltransferase 2A) histone methyltransferase complex on distinct homeobox (HOX) genes, including the osteogenic master regulator distalless homeobox 3 (DLX3) (Nayak et al., 2017). Via the deSUMOylation of RB Binding Protein 5 (RbBP5), SENP3 activates the recruitment of Ash2 (absent, small, or homeotic)-like (Ash2L) and menin subunits to DLX3 by complexing with $\operatorname{MLL1} / 2$, which is a prerequisite for promoting transcriptional activation of the HOX genes (Nayak et al., 2017).
However, the deSUMOylation of the complex of transducin $\beta$-like protein 1 (TBL1) and TBL1related 1 (TBLR1) by SENP1 decreases complex formation and subsequently inhibits $\beta$-cateninmediated transcription, serving as a suppressor of the Wnt signalling pathway (Choi et al., 2011).

\section{Macromolecular assembly}

The biogenesis of pre-ribosomal particles in eukaryotic cells is controlled by Ulp/SENPs, which was first found in S. cerevisiae via mutations in UBC9, Ulp1, and Smt3, where the export of the pre-60S ribosomal subunit was defective (Panse et al., 2006). Formed in the nucleolus, pre-60S and pre-40S ribosomes need to undergo a series of sophisticated modifications to transform them into a mature state. Subsequently, they are transported to the cytosol, in which SUMOylation plays a major role. The late steps of nucleolar maturation of pre-60S particles involves the formation of a complex comprising proline, glutamate and leucine rich protein 1 (PELP1), testis expressed 10 (TEX10), and WD Repeat Domain 18 (WDR18), the SUMOylation of which is carried out in a SENP3-dependent way (Finkbeiner et al., 2011). PELP1 shows a prominent sensitivity to SENP3, while its SUMO conjugation enhances the recruitments Midasin AAA ATPase 1 (MDN1) to pre-60S particles, functioning as a key step in pre-60S remodelling (Raman et al., 2016).

Another instructive example is that of the PML protein, a scaffold protein of PML nuclear bodies (Uversky, 2017). Being the focal point of SUMO conjugation and deconjugation, the modulation of PML nuclear bodies involves multiple SUMO-SIM interactions (Raman et al., 2013). SUMOylated PML proteins can be self-assembled via their own SIMs or recruited with other SIM-containing 
proteins. Therefore, the degree of SUMOylation greatly affects the number and composition of nuclear bodies. The key role played by SUMO in promoting nuclear bodies assembly by providing multivalent interactions was highlighted recently, suggesting the possible effect on the dynamics of nuclear bodies of SENPs (Banani et al., 2016). The poly-SUMOylated PML protein itself is a substrate for SENP6; therefore, down-regulation of SENP6 expression directly induces the formation of the SUMO chain on PML, causing an increase in both the number and size of PML nuclear bodies (Hattersley et al., 2011).

\section{DNA repair}

The importance of SUMO-dependent recruitment to the sites of DNA damage sites in the doublestrand break (DSB) response is evident from the appearance of the $70-\mathrm{kDa}$ subunit of the replication protein A complex (RPA70) being controlled by SENP6 (Dou et al., 2010). RPA plays a key role in DNA replication, as well in damage responses. Associated with RPA70 during replication, SENP6 limits the SUMOylation of RPA70 to a lower level in S phase. Double-stranded DNA damage induces the expression of the replication stressinduced factor Camptothecin (CPT), at which point SENP6 is dissociated from RPA70, thereby relieving the restriction of RPA70 SUMOylation, which involves SUMO2/3 (Dou et al., 2010). SUMOylated RPA70 recruits RAD51 to the DNA damage foci and subsequently initiates DNA repair through homologous recombination. In another example, SENP7 acts on the chromatin repressive KRAB-associated protein 1 (KAP1), facilitating the removal of its coupling to SUMO2/3 (Garvin et al., 2013). The deSUMOylation of KAP1 contributes to chromatin relaxation through interactions between chromatin remodeller CHD3 and chromatin, which establishes the permissive chromatin environment required for DNA repair.

\section{Mitochondrial dynamics}

Previously, many proteins involved in the control of mitochondrial dynamics in mammalian cells were identified, including dynamin-related protein 1 (DRP1), a mitochondrial fission GTPase that is a substrate of SUMO1. The overexpression of SUMO1 protects DRP1 from degradation and subsequently leads to increased mitochondrial fragmentation (Harder et al., 2004). However, SENP5 can reverse this SUMO1-induced fragmentation, while silencing of its expression altered mitochondrial morphology and inhibited mitochondrial fusion (Zunino et al., 2007). Moreover, the translocation of SENP5 at G2/M also has a crucial role in the regulation of DRP1-dependent fusion during mitosis (Gong and Yeh, 2006).

\section{DeSUMOylation in diseases}

As one of the most dominant post-translational modification, the substrates of SUMOylation are involved in almost all pathological processes. Thus, abnormal SUMOylation, especially the alteration of SENPs expression under diseased states, may be closely related to the development of various diseases, such as cancers and cardiac disorders. For example, SENP2, which is one of the direct targets of the transcription factor NF- $\mathrm{kB}$, accelerates the pathogenesis of tumours via inflammatory signalling (Huang et al., 2003; Lee et al., 2011). SENP3 coupled with SENP5 are notably overexpressed in oral squamous cell carcinomas, osteosarcoma, and hepatocellular carcinoma (Ding et al., 2008; Sun et al., 2013; Wang and Zhang, 2014; Jin et al., 2016). In addition, up-regulated SENP3/SMT3IP1 promotes epithelial ovarian cancer progression; thus, SENP3/SMT3IP1 up-regulation could be regarded as a novel biomarker for prognosis (Cheng et al., 2017). Moreover, the statistical relation between the expression level of SENP5 and prognosis in patients with breast cancer has been demonstrated (Cashman et al., 2014).

In addition, recent studies have also associated SUMOylation with the development, metabolism, and pathology of the heart. Numerous key proteins in cardiac development have been shown to undergo SUMOylation, including myocardin, GATA-binding protein (GATA)-4, Nk2 homeobox 5 (Nkx2.5), myocyte enhancer factor-2 (MEF2), and T-box transcription factors-2 and -5 (TBX2/ TBX5) (Wang and Schwartz, 2010; Beketaev et al., 2014). Meanwhile, SUMO elements are indispensable to the entire cardiac physiology. For instance, the absence of SENP2 resulted in cardiac hypoplasia in mice, whereas its overexpression was associated with cardiac dysfunction, such as congenital heart defects, cardiomyopathy, and hypertrophy (Kang et al., 2010; Kim et al., 2012). 


\section{Advances in the development of SENP inhibitors}

\section{Small molecule inhibitors}

To date, relatively few inhibitors of SENPs have been reported, and they are mainly concentrated in inhibiting SENP1 and SENP2. Several methods have been used to identify SENP inhibitors. Scientists used the feature that the cysteine on SENP can react with electrophiles (Hemelaar et al., 2004), and developed covalent inhibitors for this series of protein. Some researchers simulated the structure of SUMO (Albrow et al., 2011; Ponder et al., 2011), while other simulated the combination between SUMO and SENP, and utilized computer-aided drug design to develop relevant inhibitors (Qiao et al., 2011). Moreover, with the advances in computer technology, more and more research groups began to use in silico techniques to find compounds with high activity and selectivity from large libraries of compounds (Shen et al., 2006; Chen et al., 2012; Madu et al., 2013; Kumar et al., 2014; Wen et al., 2014). Non-covalent inhibitors of SENP have also been found using this method (Chen et al., 2012).

In 2011, Bogyo's (Albrow et al., 2011; Ponder et al., 2011) and Zhou's (Qiao et al., 2011) laboratories first identified small molecule inhibitors of SENPs. Bogyo's group developed a series of compounds that simulated the structure of peptides, while Zhou's group developed the first series of non-peptide inhibitors of SENPs.

During functional studies of Plasmodium falciparum SENPs, Ponder et al. (2011) screened 508 irreversible cysteine protease inhibitors, and identified a PfSENP1 inhibitor 1. PfSENP1 inhibitor 1 displayed an $\mathrm{IC}_{50}$ of $17.9 \mu \mathrm{M}$ for PfSENP1, but the values were only $9.0 \mu \mathrm{M}$ and $4.7 \mu \mathrm{M}$ for human SENP1 and SENP2, respectively. To improve the stability of the compound, as well as simplify its synthesis, the aspartic acid side chain of the original compound was removed to form compound 2. Compound 2 showed increased inhibitory efficiency. For PfSENP1, the $\mathrm{IC}_{50}$ value was $16.2 \mu \mathrm{M}$, while for human SENP1 and SENP2, the value values were $7.1 \mu \mathrm{M}$ and $3.7 \mu \mathrm{M}$, respectively. AS part of Bogyo's group, Albrow et al. (2011) designed a series compounds with acyloxymethyl ketone (AOMK), which were based on the structure of compound 2 and SUMO. Most of the compounds showed inhibitory activities on human
SENP1 and SENP2, among which, compound 3, with the QTGG specificity sequence, showed the best inhibition and $\mathrm{IC}_{50}$ values of 3.6 and $0.25 \mu \mathrm{M}$, for human SENP1 and SENP2, respectively. However, compound 4, which contains the sequence of ubiquitin, showed inhibitory activities on human SENP6 $\left(\mathrm{IC}_{50}=4.2 \mu \mathrm{M}\right)$ and SENP7 $\left(\mathrm{IC}_{50}=4.3 \mu \mathrm{M}\right)$ (Fig. 4.4).

However, considering that the compounds with peptidyl moieties may perform poorly in pharmacokinetics, Qiao et al. (2011) developed a series of SENP1 inhibitors based on a benzodiazepine scaffold, which were the first designed and synthesized non-peptide inhibitors. According to the crystal structure of human SENP1 complexed with unprocessed SUMO1 (PDB: 2IY1) (Shen et al., 2006), they found that the core structure of benzodiazepine docked into the catalytic pocket and could simulated the natural combination, via its formyl group forming a covalent bond with Cys-603. The two most potent compounds 5 and 6 displayed IC $_{50}$ values of $19.5 \mu \mathrm{M}$ and $9.2 \mu \mathrm{M}$, respectively, for SENP1, and also showed inhibitory activity against prostate cancer cells in vitro, with $\mathrm{IC}_{50}$ of $13.0 \mu \mathrm{M}$ and $35.7 \mu \mathrm{M}$, respectively. In a follow-up study, Zhao et al. (2016) found 11 series of SENP1 inhibitors with different scaffolds using virtual screening. By analysing the structures of these inhibitors and the patterns of their binding to SENP1, a series of compounds with new scaffolds was designed and synthesized from two representative compounds. Subsequently, their structure-activity relationships were identified. Among them, the most potent compound 7 displayed an $\mathrm{IC}_{50}$ of $3.5 \mu \mathrm{M}$ for SENP1 (Fig. 4.5).

Compound 8 can inhibit hypoxia inducible factor (HIF)-1 $\alpha$ accumulation (Uno et al., 2009), as well as the growth of KEK293 cells $\left(\mathrm{IC}_{50}=7.2 \mu \mathrm{M}\right)$. However, its inhibitory mechanism has not been determined (Shimizu et al., 2010). Uno et al. (2012) used a biotin-tagged compound version of compound 8 to identify its target molecules using pull-down experiments. Fortunately, they observed an interaction between 8 and SENP1. Through structural optimization, compounds 9 and 10 were synthesized, which have more potent inhibitory activities against SENP1, with $\mathrm{IC}_{50}$ values of $39.5 \mu \mathrm{M}$ and $29.6 \mu \mathrm{M}$, respectively (Fig. 4.6).

In recent years, computational approaches have become important to identify small molecule 
<smiles></smiles>

1<smiles>CC(C)C(NC(=O)[C@H](CCC(N)=O)NC(=O)Cc1ccc(O)c([N+](=O)[O-])c1)C(=O)NCC(=O)NCC(=O)COC(=O)c1c2ccccc2cc2ccccc12</smiles>

3

Figure 4.4 Structures of compounds 1-4.<smiles></smiles>

2<smiles>CC(C)C[C@H](NC(=O)Cc1ccc(O)c([N+](=O)[O-])c1)C(=O)NC(CCCNC(=N)N)C(=O)NCC(=O)NCC(=O)COC(=O)c1c2ccccc2cc2ccccc12</smiles>

4

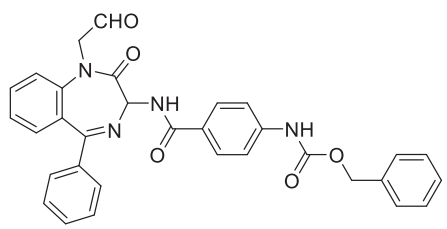

5

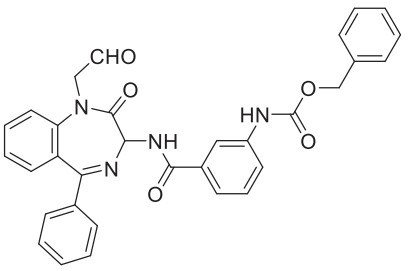

6<smiles>COc1ccc(-c2cccc(C(=O)NCc3cccc(NC(=O)CCc4ccccc4)c3)c2)cc1</smiles>

Figure 4.5 Structures of compounds 5-7.

inhibitors. Several approaches have been reported, such as virtual screening and docking, in attempts to find SENPs inhibitors.

Based on the crystal structure of the SENP1SUMO2-RanGAP1 complex reported by Hay's group (PDB entry: 2IY0) (Shen et al., 2006), Chen et al. (2012) identified novel lead compound 11 as a SENP1 inhibitor by molecular docking of 180,000 compounds in the SPECS compound library using Glide version 4.5. According to the results of subsequent biological tests of the selected 38 compounds, compound 11 showed the best inhibitory activity, with an $\mathrm{IC}_{50}$ of $2.385 \mu \mathrm{M}$. A series of derivatives of 11 based on 2-(4-Chlorophenyl)-2-oxoethyl 4-benzamidobenzoate was then designed and synthesized, among which the $\mathrm{IC}_{50}$ of compound 12 reached $1.08 \mu \mathrm{M}$ (Fig. 4.7).

Madu et al. (2013) performed in silico screening of 250,000 compounds using the program GLIDE and obtained 40 candidates that exhibited inhibitory activities on SENP1, SENP2, and SENP7. According to the data from biological measurement and their structural features, a novel class of SENP inhibitors based on sulfonyl-benzene groups was proposed. The most potent compound 13 displayed $\mathrm{IC}_{50}$ values of $2.1 \mu \mathrm{M}, 2.0 \mu \mathrm{M}$, and $2.7 \mu \mathrm{M}$, respectively, for SENP1, SENP2, and SENP7. Moreover, unlike the most common SENP inhibitors, which 


$$
8\left(\mathrm{R}^{1}=\mathrm{H}, \mathrm{R}^{2}=\mathrm{CH}_{3}\right) ; 9\left(\mathrm{R}^{1}=\mathrm{CH}_{3}, \mathrm{R}^{2}=\mathrm{CH}_{3}\right) ; \mathbf{1 0}\left(\mathrm{R}^{1}=\mathrm{CH}_{3}, \mathrm{R}^{2}=\mathrm{C}_{2} \mathrm{H}_{5}\right)
$$

Figure 4.6 Structures of compounds 8-10.<smiles>COc1cccc(C(=O)Nc2ccc(C(=O)OCC(=O)c3ccc(Cl)cc3)cc2)c1</smiles>

11<smiles>O=C(COC(=O)c1ccc(NC(=O)c2cccc(OCc3ccccc3)c2)cc1)c1ccc(Cl)cc1</smiles>

12

Figure 4.7 Structures of compounds 11-12.

covalently target the catalytic cysteine residue, this class of inhibitors are not covalently bound to SENPs. They have a non-competitive inhibitory mechanism and can combine with SENPs and the SENPs-substrates complex (Fig. 4.8).

Kumar et al. (2014) screened out small molecules from a library of 400 million compounds using the ROCS and EON programs. The molecules were similar to the TGGK peptide at the SUMO1 C-terminus in terms of their structure and electrostatic characteristics. These compounds were docked to SENP2 catalytic pocket and then a quantitative biological test was performed on the selected 49 compounds using oxadiazole. This series of compounds showed inhibitory activities on both SENP1 and SENP2. Finally, the most potent compound 14 was found to show an $\mathrm{IC}_{50}$ of $3.7 \mu \mathrm{M}$ on SENP2 and $>30 \mu \mathrm{M}$ on SENP1, which indicated partial selectivity for SENP2 (Fig. 4.9).

Wen et al. (2014) used two virtual screening programs, DOCK and AutoDock to docked $\approx 100,000$ drug-like compounds, which were selected from a library comprising two million compounds. Finally, 117 compounds were selected to evaluate SENP1 activity. The most potent compound 15 displayed an $\mathrm{IC}_{50}$ of $1.29 \mu \mathrm{M}$ for SENP1. It shows selectivity for SENP1, but weak inhibition of other cysteine proteases, like cathepsin B and D (Fig. 4.10).

\section{Biotinylated probes}

Before these small molecules were identified, scientists focused on covalent binding with the thiol group of cysteine in the catalytic site of SENPs.

Hemelaar et al. (2004) first reported peptide SENPs inhibitors. Based on the mechanism of SUMOylation and the structural characteristics of SUMO, they used the synthesis strategy of intein to link the vinylsulfone (VS) group at the end of SUMO to obtain the peptide SENP inhibitor 16. A Michael addition reaction occurred, and the inhibitor covalently bound to the catalytic cysteine residues of SENP2 and other related enzymes. To identify the key role of the cysteine residues in catalysis, pre-incubation of SENP2 with N-ethylmaleimide (NEM) was carried out, which disrupted the irreversible conjugation between VS and SENP2 (Fig. 4.11).

Borodovsky et al. (2005) used a similar strategy and reported peptide compounds of the ubiquitin-like proteins Nedd8, SUMO1, FAT10, Fau, and APG12 linked to a VS group. Among them, there are three $\mathrm{C}$-terminal peptide chains 
<smiles>Cc1cc(-c2ccc(N=Nc3c(N)c(S(=O)(=O)O)cc4cc(C(=O)O)ccc34)c(C)c2)ccc1N=Nc1c(N)ccc2cc(C(=O)O)ccc12</smiles>

Figure 4.8 Structure of compound 13.

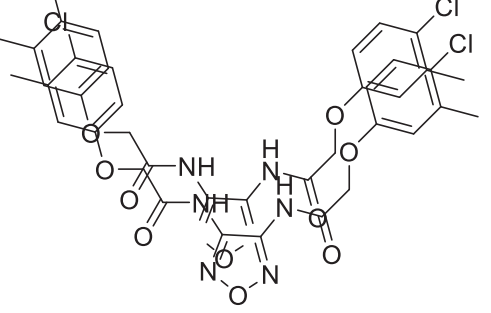

Figure 4.9 Structure of compound 14.<smiles>O=C(/C=C/C1C(OC(=O)c2ccc(-c3ccccc3)cc2)CC2OC(=O)CC21)COc1cccc(Cl)c1</smiles>

15

Figure 4.10 Structure of compound 15.

of different lengths from SUMO1 (5 peptide, 9 peptide, 13 peptide) linked to the VS group that could bind to a series of proteins in the EL-4 cell lysate. Subsequent competition experiments showed that the peptide 17 was able to bind to at least one SUMO1 protease and was sufficient to establish selectivity. This study showed that only peptides of a few amino acids can specifically bind to SENPs (Fig. 4.12).
Dobrotă et al. (2012) designed and synthesized peptide 18. One terminal of the peptide contains a glycine-derived fluoromethylketone group that can covalently bind to the cysteine of SENPs. The results showed that peptide C binds to SENP1 and SENP2 and can compete for SUMO1 from the SENP1-SUMO1 complex, indicating that this compound binds to SENP1 more strongly than the SUMO1 molecule in nature (Fig. 4.13). 
<smiles>COC(=O)NC/C=C/S(C)(=O)=O</smiles>

Figure 4.11 Structure of compound 16.

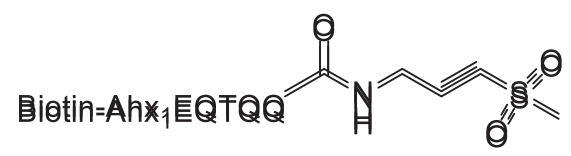

Figure 4.12 Structure of compound 17.<smiles>O=C(CF)CNC(=O)CNC(=O)[OH+]O[Se]O[GeH2]OCc1ccccc1</smiles>

Figure 4.13 Structure of compound 18.

\section{References}

Albrow, V.E., Ponder, E.L., Fasci, D., Békés, M., Deu, E., Salvesen, G.S., and Bogyo, M. (2011). Development of small molecule inhibitors and probes of human SUMO deconjugating proteases. Chem. Biol. 18, 722-732. https://doi.org/10.1016/j.chembiol.2011.05.008.

Alegre, K.O., and Reverter, D. (2011). Swapping small ubiquitin-like modifier (SUMO) isoform specificity of SUMO proteases SENP6 and SENP7. J. Biol. Chem. 286, 36142-36151. https://doi.org/10.1074/jbc. M111.268847.

Bailey, D., and O'Hare, P. (2004). Characterization of the localization and proteolytic activity of the SUMOspecific protease, SENP1. J. Biol. Chem. 279, 692-703. https://doi.org/10.1074/jbc.M306195200.

Bailey, M., Srivastava, A., Conti, L., Nelis, S., Zhang, C., Florance, H., Love, A., Milner, J., Napier, R., Grant, M., et al. (2016). Stability of small ubiquitin-like modifier (SUMO) proteases overly tolerant to salt1 and -2 modulates salicylic acid signalling and SUMO1/2 conjugation in Arabidopsis thaliana. J. Exp. Bot. 67, 353-363. https://doi.org/10.1093/jxb/erv468.

Banani, S.F., Rice, A.M., Peeples, W.B., Lin, Y., Jain, S., Parker, R., and Rosen, M.K. (2016). Compositional control of phase-separated cellular bodies. Cell 166, 651-663.

Békés, M., Prudden, J., Srikumar, T., Raught, B., Boddy, M.N., and Salvesen, G.S. (2011). The dynamics and mechanism of SUMO chain deconjugation by SUMOspecific proteases. J. Biol. Chem. 286, 10238-10247. https://doi.org/10.1074/jbc.M110.205153.
Beketaev, I., Kim, E.Y., Zhang, Y., Yu, W., Qian, L., and Wang, J. (2014). Potentiation of Tbx5-mediated transactivation by SUMO conjugation and protein inhibitor of activated STAT 1 (PIAS1). Int. J. Biochem. Cell Biol. 50, 82-92. https://doi.org/10.1016/j.biocel.2014.02.007.

Best, J.L., Ganiatsas, S., Agarwal, S., Changou, A., Salomoni, P., Shirihai, O., Meluh, P.B., Pandolfi, P.P., and Zon, L.I. (2002). SUMO-1 protease-1 regulates gene transcription through PML. Mol. Cell 10, 843-855.

Borodovsky, A., Ovaa, H., Meester, W.J., Venanzi, E.S., Bogyo, M.S., Hekking, B.G., Ploegh, H.L., Kessler, B.M., and Overkleeft, H.S. (2005). Small-molecule inhibitors and probes for ubiquitin- and ubiquitin-like-specific proteases. Chembiochem 6, 287-291. https://doi. org/10.1002/cbic.200400236.

Cappadocia, L., Pichler, A., and Lima, C.D. (2015). Structural basis for catalytic activation by the human ZNF451 SUMO E3 ligase. Nat. Struct. Mol. Biol. 22, 968-975. https://doi.org/10.1038/nsmb.3116.

Cashman, R., Cohen, H., Ben-Hamo, R., Zilberberg, A., and Efroni, S. (2014). SENP5 mediates breast cancer invasion via a TGF $\beta$ RI SUMOylation cascade. Oncotarget 5, 1071-1082.

Castro, P.H., Couto, D., Freitas, S., Verde, N., Macho, A.P., Huguet, S., Botella, M.A., Ruiz-Albert, J., Tavares, R.M., Bejarano, E.R., et al. (2016). SUMO proteases ULP1c and ULP1d are required for development and osmotic stress responses in Arabidopsis thaliana. Plant Mol. Biol. 92, 143-159. https://doi.org/10.1007/s11103-0160500-9. 
Chen, Y., Wen, D., Huang, Z., Huang, M., Luo, Y., Liu, B., Lu, H., Wu, Y., Peng, Y., and Zhang, J. (2012). 2-(4-Chlorophenyl)-2-oxoethyl 4-benzamidobenzoate derivatives, a novel class of SENP1 inhibitors: Virtual screening, synthesis and biological evaluation. Bioorg. Med. Chem. Lett. 22, 6867-6870. https://doi. org/10.1016/j.bmcl.2012.09.037.

Cheng, J., Perkins, N.D., and Yeh, E.T. (2005). Differential regulation of c-Jun-dependent transcription by SUMOspecific proteases. J. Biol. Chem. 280, 14492-14498.

Cheng, J., Su, M., Jin, Y., Xi, Q. Deng, Y., Chen, J., Wang, W., Chen, Y., Chen, L., Shi, N., et al. (2017). Upregulation of SENP3/SMT3IP1 promotes epithelial ovarian cancer progression and forecasts poor prognosis. Tumour Biol. 39, 1010428317694543. https://doi. org/10.1177/1010428317694543.

Choi, H.K., Choi, K.C., Yoo, J.Y., Song, M., Ko, S.J., Kim, C.H., Ahn, J.H., Chun, K.H., Yook, J.I., and Yoon, H.G. (2011). Reversible SUMOylation of TBL1TBLR1 regulates $\beta$-catenin-mediated Wnt signaling. Mol. Cell 43, 203-216. https://doi.org/10.1016/j. molcel.2011.05.027.

Conti, L., Nelis, S., Zhang, C., Woodcock, A., Swarup, R., Galbiati, M., Tonelli, C., Napier, R., Hedden, P., Bennett, M., et al. (2014). Small Ubiquitin-like Modifier protein SUMO enables plants to control growth independently of the phytohormone gibberellin. Dev. Cell 28, 102-110. https://doi.org/10.1016/j.devcel.2013.12.004.

Cubeñas-Potts, C., Goeres, J.D., and Matunis, M.J. (2013). SENP1 and SENP2 affect spatial and temporal control of sumoylation in mitosis. Mol. Biol. Cell 24, 3483-3495. https://doi.org/10.1091/mbc.E13-05-0230.

Danielsen, J.R., Povlsen, L.K., Villumsen, B.H., Streicher, W., Nilsson, J., Wikström, M., Bekker-Jensen, S., and Mailand, N. (2012). DNA damage-inducible SUMOylation of HERC2 promotes RNF8 binding via a novel SUMO-binding Zinc finger. J. Cell Biol. 197, 179-187. https://doi.org/10.1083/jcb.201106152.

Di Bacco, A., Ouyang, J., Lee, H.Y., Catic, A., Ploegh, H., and Gill, G. (2006). The SUMO-specific protease SENP5 is required for cell division. Mol. Cell. Biol. 26, 4489-4498.

Ding, X., Sun, J., Wang, L., Li, G., Shen, Y., Zhou, X., and Chen, W. (2008). Overexpression of SENP5 in oral squamous cell carcinoma and its association with differentiation. Oncol. Rep. 20, 1041-1045.

Dobrotă, C., Fasci, D., Hădade, N.D., Roiban, G.D., Pop, C., Meier, V.M., Dumitru, I., Matache, M., Salvesen, G.S., and Funeriu, D.P. (2012). Glycine fluoromethylketones as SENP-specific activity based probes. Chembiochem 13, 80-84. https://doi.org/10.1002/cbic.201100645.

Dou, H., Huang, C., Singh, M., Carpenter, P.B., and Yeh, E.T. (2010). Regulation of DNA repair through deSUMOylation and SUMOylation of replication protein A complex. Mol. Cell 39, 333-345. https://doi. org/10.1016/j.molcel.2010.07.021.

Enserink, J.M. (2015). Sumo and the cellular stress response. Cell Div. 10, 4. https://doi.org/10.1186/ s13008-015-0010-1.

Finkbeiner, E., Haindl, M., Raman, N., and Muller, S. (2011). SUMO routes ribosome maturation. Nucleus 2, 527-532. https://doi.org/10.4161/nucl.2.6.17604.

Galanty, Y., Belotserkovskaya, R., Coates, J., and Jackson, S.P. (2012). RNF4, a SUMO-targeted ubiquitin E3 ligase, promotes DNA double-strand break repair. Genes Dev. 26, 1179-1195. https://doi.org/10.1101/ gad.188284.112.

Garvin, A.J., Densham, R.M., Blair-Reid, S.A., Pratt, K.M., Stone, H.R., Weekes, D., Lawrence, K.J., and Morris, J.R. (2013). The deSUMOylase SENP7 promotes chromatin relaxation for homologous recombination DNA repair. EMBO Rep. 14, 975-983. https://doi. org/10.1038/embor.2013.141.

Gill, G. (2005). Something about SUMO inhibits transcription. Curr. Opin. Genet. Dev. 15, 536-541.

Goeres, J., Chan, P.K., Mukhopadhyay, D., Zhang, H., Raught, B., and Matunis, M.J. (2011). The SUMOspecific isopeptidase SENP2 associates dynamically with nuclear pore complexes through interactions with karyopherins and the Nup107-160 nucleoporin subcomplex. Mol. Biol. Cell 22, 4868-4882. https:// doi.org/10.1091/mbc.E10-12-0953.

Gong, L., and Yeh, E.T. (2006). Characterization of a family of nucleolar SUMO-specific proteases with preference for SUMO-2 or SUMO-3. J. Biol. Chem. 281, 15869 15877.

Guo, D., Li, M., Zhang, Y., Yang, P., Eckenrode, S., Hopkins, D., Zheng, W., Purohit, S., Podolsky, R.H., Muir, A., et al. (2004). A functional variant of SUMO4, a new I kappa $\mathrm{B}$ alpha modifier, is associated with type 1 diabetes. Nat. Genet. 36, 837-841. https://doi.org/10.1038/ng1391.

Guzzo, C.M., Ringel, A., Cox, E., Uzoma, I., Zhu, H., Blackshaw, S., Wolberger, C., and Matunis, M.J. (2014). Characterization of the SUMO-binding activity of the myeloproliferative and mental retardation (MYM)type zinc fingers in ZNF261 and ZNF198. PLOS ONE 9, e105271. https://doi.org/10.1371/journal. pone. 0105271 .

Haindl, M., Harasim, T., Eick, D., and Muller, S. (2008). The nucleolar SUMO-specific protease SENP3 reverses SUMO modification of nucleophosmin and is required for rRNA processing. EMBO Rep. 9, 273-279. https:// doi.org/10.1038/embor.2008.3.

Harder, Z., Zunino, R., and McBride, H. (2004). Sumo1 conjugates mitochondrial substrates and participates in mitochondrial fission. Curr. Biol. 14, 340-345. https:// doi.org/10.1016/j.cub.2004.02.004.

Hattersley, N., Shen, L., Jaffray, E.G., and Hay, R.T. (2011). The SUMO protease SENP6 is a direct regulator of PML nuclear bodies. Mol. Biol. Cell 22, 78-90. https://doi. org/10.1091/mbc.E10-06-0504.

Hemelaar, J., Borodovsky, A., Kessler, B.M., Reverter, D., Cook, J., Kolli, N., Gan-Erdene, T., Wilkinson, K.D., Gill, G., Lima, C.D., et al. (2004). Specific and covalent targeting of conjugating and deconjugating enzymes of ubiquitin-like proteins. Mol. Cell. Biol. 24, 84-95.

Hendriks, I.A., and Vertegaal, A.C. (2016). A comprehensive compilation of SUMO proteomics. Nat. Rev. Mol. Cell Biol. 17, 581-595. https://doi.org/10.1038/ nrm.2016.81.

Hickey, C.M., Wilson, N.R., and Hochstrasser, M. (2012). Function and regulation of SUMO proteases. Nat. Rev. Mol. Cell Biol. 13, 755-766. https://doi.org/10.1038/ nrm3478.

Hietakangas, V., Ahlskog, J.K., Jakobsson, A.M., Hellesuo, M., Sahlberg, N.M., Holmberg, C.I., Mikhailov, A., Palvimo, J.J., Pirkkala, L., and Sistonen, L. (2003). 
Phosphorylation of serine 303 is a prerequisite for the stress-inducible SUMO modification of heat shock factor 1. Mol. Cell. Biol. 23, 2953-2968.

Huang, T.T., Wuerzbrger-Davis, S.M., Wu, Z.H., and Miyamoto, S. (2003). Sequential modification of NEMO/IKK gamma by SUMO-1 and ubiquitin mediates NF-kappa B activation by genotoxic stress. Cell 115, 565-576. http://dx.doi.org/10.1016/s00928674(03)00895-x.

Itahana, Y., Yeh, E.T., and Zhang, Y. (2006). Nucleocytoplasmic shuttling modulates activity and ubiquitination-dependent turnover of SUMO-specific protease 2. Mol. Cell. Biol. 26, 4675-4689.

Jardin, C., Horn, A.H., and Sticht, H. (2015). Binding properties of SUMO-interacting motifs (SIMs) in yeast. J. Mol. Model. 21, 50. https://doi.org/10.1007/s00894015-2597-1.

Jentsch, S., and Psakhye, I. (2013). Control of nuclear activities by substrate-selective and protein-group SUMOylation. Annu. Rev. Genet. 47, 167-186. https:// doi.org/10.1146/annurev-genet-111212-133453.

Jin, Z.L., Pei, H., Xu, Y.H., Yu, J., and Deng, T. (2016). The SUMO-specific protease SENP5 controls DNA damage response and promotes tumorigenesis in hepatocellular carcinoma. Eur. Rev. Med. Pharmacol. Sci. 20, 3566 3573.

Johnson, E.S. (2004). Protein modification by SUMO. Annu. Rev. Biochem. 73, 355-382. http://dx.doi. org/10.1146/annurev.biochem.73.011303.074118.

Kang, X., Qi, Y., Zuo, Y., Wang, Q. Zou, Y., Schwartz, R.J., Cheng, J., and Yeh, E.T. (2010). SUMO-specific protease 2 is essential for suppression of polycomb group protein-mediated gene silencing during embryonic development. Mol. Cell 38, 191-201. https://doi. org/10.1016/j.molcel.2010.03.005.

Kerscher, O., Felberbaum, R., and Hochstrasser, M. (2006). Modification of proteins by ubiquitin and ubiquitin-like proteins. Annu. Rev. Cell Dev. Biol.22, 159-180.https:// doi.org/10.1146/annurev.cellbio.22.010605.093503.

Kim, E.Y., Chen, L., Ma, Y., Yu, W., Chang, J., Moskowitz, I.P., and Wang, J. (2012). Enhanced desumoylation in murine hearts by overexpressed SENP2 leads to congenital heart defects and cardiac dysfunction. J. Mol. Cell. Cardiol. 52, 638-649. https://doi.org/10.1016/j. yjmcc.2011.11.011.

Knipscheer, P., Flotho, A., Klug, H., Olsen, J.V., van Dijk, W.J., Fish, A., Johnson, E.S., Mann, M., Sixma, T.K., and Pichler, A. (2008). Ubc9 sumoylation regulates SUMO target discrimination. Mol. Cell 31, 371-382. https:// doi.org/10.1016/j.molcel.2008.05.022.

Kumar, A., and Zhang, K.Y. (2015). Advances in the development of SUMO specific protease (SENP) inhibitors. Comput. Struct. Biotechnol. J. 13, 204-211. https://doi.org/10.1016/j.csbj.2015.03.001.

Kumar, A., Ito, A., Takemoto, M., Yoshida, M., and Zhang, K.Y. (2014). Identification of 1,2,5-oxadiazoles as a new class of SENP2 inhibitors using structure based virtual screening. J. Chem. Inf. Model. 54, 870-880. https:// doi.org/10.1021/ci4007134.

Kung, C.C., Naik, M.T., Wang, S.H., Shih, H.M., Chang, C.C., Lin, L.Y., Chen, C.L., Ma, C., Chang, C.F., and Huang, T.H. (2014). Structural analysis of poly-SUMO chain recognition by the RNF4-SIMs domain. Biochem. J. 462, 53-65. https://doi.org/10.1042/BJ20140521.

Lee, M.H., Mabb, A.M., Gill, G.B., Yeh, E.T., and Miyamoto, S. (2011). NF- $\kappa B$ induction of the SUMO protease SENP2: A negative feedback loop to attenuate cell survival response to genotoxic stress. Mol. Cell 43, 180191. https://doi.org/10.1016/j.molcel.2011.06.017.

Li, S.J., and Hochstrasser, M. (1999). A new protease required for cell-cycle progression in yeast. Nature 398, 246-251. https://doi.org/10.1038/18457.

Li, S.J., and Hochstrasser, M. (2000). The yeast ULP2 (SMT4) gene encodes a novel protease specific for the ubiquitin-like Smt3 protein. Mol. Cell. Biol. 20, 2367-2377.

Lima, C.D., and Reverter, D. (2008). Structure of the human SENP7 catalytic domain and poly-SUMO deconjugation activities for SENP6 and SENP7. J. Biol. Chem. 283, 32045-32055. https://doi.org/10.1074/ jbc.M805655200.

Lin, D., Tatham, M.H., Yu, B., Kim, S., Hay, R.T., and Chen, Y. (2002). Identification of a substrate recognition site on Ubc9. J. Biol. Chem. 277, 21740-21748. https://doi. org/10.1074/jbc.M108418200.

Liu, Y., Kieslich, C.A., Morikis, D., and Liao, J. (2014). Engineering pre-SUMO4 as efficient substrate of SENP2. Protein Eng. Des. Sel. 27, 117-126. https://doi. org/10.1093/protein/gzu004.

Madu, I.G., Namanja, A.T., Su, Y., Wong, S., Li, Y.J., and Chen, Y. (2013). Identification and characterization of a new chemotype of noncovalent SENP inhibitors. ACS Chem. Biol. 8, 1435-1441. https://doi.org/10.1021/ cb400177q.

Mahajan, R., Delphin, C., Guan, T., Gerace, L., and Melchior, F. (1997). A small ubiquitin-related polypeptide involved in targeting RanGAP1 to nuclear pore complex protein RanBP2. Cell 88, 97-107.

Matic, I., Schimmel, J., Hendriks, I.A., van Santen, M.A., van de Rijke, F., van Dam, H., Gnad, F., Mann, M., and Vertegaal, A.C. (2010). Site-specific identification of SUMO-2 targets in cells reveals an inverted SUMOylation motif and a hydrophobic cluster SUMOylation motif. Mol. Cell 39, 641-652. https:// doi.org/10.1016/j.molcel.2010.07.026.

Meluh, P.B., and Koshland, D. (1995). Evidence that the MIF2 gene of Saccharomyces cerevisiae encodes a centromere protein with homology to the mammalian centromere protein CENP-C. Mol. Biol. Cell 6, 793807.

Merrill, J.C., Melhuish, T.A., Kagey, M.H., Yang, S.H., Sharrocks, A.D., and Wotton, D. (2010). A role for noncovalent SUMO interaction motifs in Pc2/CBX4 E3 activity. PLOS ONE 5, e8794. https://doi.org/10.1371/ journal.pone.0008794.

Meulmeester, E., Kunze, M., Hsiao, H.H., Urlaub, H., and Melchior, F. (2008). Mechanism and consequences for paralog-specific sumoylation of ubiquitin-specific protease 25. Mol. Cell 30, 610-619. https://doi. org/10.1016/j.molcel.2008.03.021.

Miura, K., and Hasegawa, P.M. (2010). Sumoylation and other ubiquitin-like post-translational modifications in plants. Trends Cell Biol. 20, 223-232. https://doi. org/10.1016/j.tcb.2010.01.007. 
Mohideen, F., Capili, A.D., Bilimoria, P.M., Yamada, T., Bonni, A., and Lima, C.D. (2009). A molecular basis for phosphorylation-dependent SUMO conjugation by the E2 UBC9. Nat. Struct. Mol. Biol. 16, 945-952. https:// doi.org/10.1038/nsmb.1648.

Mukhopadhyay, D., and Dasso, M. (2007). Modification in reverse: the SUMO proteases. Trends Biochem. Sci. 32, 286-295.

Mukhopadhyay, D., and Dasso, M. (2010). The fate of metaphase kinetochores is weighed in the balance of SUMOylation during S phase. Cell Cycle 9, 3194-3201. https://doi.org/10.4161/cc.9.16.12619.

Nayak, A., Reck, A., Morsczeck, C., and Mueller, S. (2017). Flightless-I governs cell fate by recruiting the SUMO isopeptidase SENP3 to distinct HOX genes. Epigenetics Chromatin 10, 15. https://doi.org/10.1186/s13072017-0122-8.

Neyret-Kahn, H., Benhamed, M., Ye, T., Le Gras, S., Cossec, J.C., Lapaquette, P., Bischof, O., Ouspenskaia, M., Dasso, M., Seeler, J., et al. (2013). Sumoylation at chromatin governs coordinated repression of a transcriptional program essential for cell growth and proliferation. Genome Res. 23, 1563-1579. https://doi.org/10.1101/ gr.154872.113.

Nishida, T., Tanaka, H., and Yasuda, H. (2000). A novel mammalian Smt3-specific isopeptidase 1 (SMT3IP1) localized in the nucleolus at interphase. Eur. J. Biochem. 267, 6423-6427.

Niskanen, E.A., Malinen, M., Sutinen, P., Toropainen, S., Paakinaho, V., Vihervaara, A., Joutsen, J., Kaikkonen, M.U., Sistonen, L., and Palvimo, J.J. (2015). Global SUMOylation on active chromatin is an acute heat stress response restricting transcription. Genome Biol. 16, 153. https://doi.org/10.1186/s13059-015-0717-y.

Niskanen, E.A., and Palvimo, J.J. (2017). Chromatin SUMOylation in heat stress: To protect, pause and organise?: SUMO stress response on chromatin. Bioessays 39,. https://doi.org/10.1002/ bies.201600263.

Owerbach, D., McKay, E.M., Yeh, E.T., Gabbay, K.H., and Bohren, K.M. (2005). A proline-90 residue unique to SUMO-4 prevents maturation and sumoylation. Biochem. Biophys. Res. Commun. 337, 517-520.

Panse, V.G., Kressler, D., Pauli, A., Petfalski, E., Gnädig, M., Tollervey, D., and Hurt, E. (2006). Formation and nuclear export of preribosomes are functionally linked to the small-ubiquitin-related modifier pathway. Traffic 7, 1311-1321.

Park, H.J., Kim, W.Y., Park, H.C., Lee, S.Y., Bohnert, H.J., and Yun, D.J. (2011). SUMO and SUMOylation in plants. Mol. Cells 32, 305-316. https://doi.org/10.1007/ s10059-011-0122-7.

Pichler, A., Knipscheer, P., Oberhofer, E., van Dijk, W.J., Körner, R., Olsen, J.V., Jentsch, S., Melchior, F., and Sixma, T.K. (2005). SUMO modification of the ubiquitin-conjugating enzyme E2-25K. Nat. Struct. Mol. Biol. 12, 264-269.

Ponder, E.L., Albrow, V.E., Leader, B.A., Békés, M., Mikolajczyk, J., Fonović, U.P., Shen, A., Drag, M., Xiao, J., Deu, E., et al. (2011). Functional characterization of a SUMO deconjugating protease of Plasmodium falciparum using newly identified small molecule inhibitors. Chem. Biol. 18, 711-721. https://doi. org/10.1016/j.chembiol.2011.04.010.

Qiao, Z., Wang, W., Wang, L., Wen, D., Zhao, Y., Wang, Q. Meng, Q. Chen, G., Wu, Y., and Zhou, H. (2011). Design, synthesis, and biological evaluation of benzodiazepine-based SUMO-specific protease 1 inhibitors. Bioorg. Med. Chem. Lett. 21, 6389-6392. https://doi.org/10.1016/j.bmcl.2011.08.101.

Raman, N., Nayak, A., and Muller, S. (2013). The SUMO system: a master organizer of nuclear protein assemblies. Chromosoma 122, 475-485. https://doi.org/10.1007/ s00412-013-0429-6.

Raman, N., Weir, E., and Müller, S. (2016). The AAA ATPase MDN1 acts as a SUMO-targeted regulator in mammalian pre-ribosome remodeling. Mol. Cell 64, 607-615.

Rodriguez, M.S., Dargemont, C., and Hay, R.T. (2001). SUMO-1 conjugation in vivo requires both a consensus modification motif and nuclear targeting. J. Biol. Chem. 276, 12654-12659. https://doi.org/10.1074/jbc. M009476200.

Saracco, S.A., Miller, M.J., Kurepa, J., and Vierstra, R.D. (2007). Genetic analysis of SUMOylation in Arabidopsis: conjugation of SUMO1 and SUMO2 to nuclear proteins is essential. Plant Physiol. 145, 119-134.

Sekiyama, N., Ikegami, T., Yamane, T., Ikeguchi, M., Uchimura, Y., Baba, D., Ariyoshi, M., Tochio, H., Saitoh, H., and Shirakawa, M. (2008). Structure of the small ubiquitin-like modifier (SUMO)-interacting motif of MBD1-containing chromatin-associated factor 1 bound to SUMO-3. J. Biol. Chem. 283, 35966-35975. https:// doi.org/10.1074/jbc.M802528200.

Shen, L., Tatham, M.H., Dong, C., Zagórska, A., Naismith, J.H., and Hay, R.T. (2006). SUMO protease SENP1 induces isomerization of the scissile peptide bond. Nat. Struct. Mol. Biol. 13, 1069-1077.

Shimizu, K., Maruyama, M., Yasui, Y., Minegishi, H., Ban, H.S., and Nakamura, H. (2010). Boron-containing phenoxyacetanilide derivatives as hypoxia-inducible factor (HIF)-1 alpha inhibitors. Bioorg. Med. Chem. Lett. 20, 1453-1456. http://dx.doi.org/10.1016/j. bmcl.2009.12.037.

Stehmeier, P., and Muller, S. (2009). Phospho-regulated SUMO interaction modules connect the SUMO system to CK2 signaling. Mol. Cell 33, 400-409. https://doi. org/10.1016/j.molcel.2009.01.013.

Sun, Z., Hu, S., Luo, Q. Ye, D., Hu, D., and Chen, F. (2013). Overexpression of SENP3 in oral squamous cell carcinoma and its association with differentiation. Oncol. Rep. 29, 1701-1706. https://doi.org/10.3892/ or.2013.2318.

Uno, M., Ban, H.S., and Nakamura, H. (2009). 14-(N-Benzylamino)phenyl -3-phenylurea derivatives as a new class of hypoxia-inducible factor-1 alpha inhibitors. Bioorg. Med. Chem. Lett. 19, 3166-3169. https://doi.org/10.1016/j.bmcl.2009.04.122.

Uno, M., Koma, Y., Ban, H.S., and Nakamura, H. (2012). Discovery of 1- 4-(N-benzylamino)phenyl -3-phenylurea derivatives as non-peptidic selective SUMO-sentrin specific protease (SENP)1 inhibitors. Bioorg. Med. Chem. Lett. 22, 5169-5173. http://dx.doi. org/10.1016/j.bmcl.2012.06.084 
Uversky, V.N. (2017). Intrinsically disordered proteins in overcrowded milieu: Membrane-less organelles, phase separation, and intrinsic disorder. Curr. Opin. Struct. Biol. 44, 18-30.

Vierstra, R.D. (2012). The expanding universe of ubiquitin and ubiquitin-like modifiers. Plant Physiol. 160, 2-14. https://doi.org/10.1104/pp.112.200667.

Wang, J., and Schwartz, R.J. (2010). Sumoylation and regulation of cardiac gene expression. Circ. Res. 107, 19-29. https://doi.org/10.1161/ CIRCRESAHA.110.220491.

Wang, K., and Zhang, X.C. (2014). Inhibition of SENP5 suppresses cell growth and promotes apoptosis in osteosarcoma cells. Exp. Ther. Med. 7, 1691-1695. https://doi.org/10.3892/etm.2014.1644.

Wen, D., Xu, Z., Xia, L., Liu, X., Tu, Y., Lei, H., Wang, W., Wang, T., Song, L., Ma, C., et al. (2014). Important role of SUMOylation of Spliceosome factors in prostate cancer cells. J. Proteome Res. 13, 3571-3582. https:// doi.org/10.1021/pr4012848.

Wilkinson, K.A., and Henley, J.M. (2010). Mechanisms, regulation and consequences of protein SUMOylation. Biochem. J. 428, 133-145. https://doi.org/10.1042/ BJ20100158.

Wotton, D., Pemberton, L.F., and Merrill-Schools, J. (2017). SUMO and Chromatin Remodeling. Adv. Exp. Med. Biol. 963, 35-50. http://dx.doi.org/10.1007/978-3319-50044-7 3

$\mathrm{Xu}$, Z., Chau, S.F., Lam, K.H., Chan, H.Y., Ng, T.B., and $\mathrm{Au}$, S.W. (2006). Crystal structure of the SENP1 mutant C603S-SUMO complex reveals the hydrolytic mechanism of SUMO-specific protease. Biochem. J. 398, 345-352.

Yan, S., Sun, X., Xiang, B., Cang, H., Kang, X., Chen, Y., Li, H., Shi, G., Yeh, E.T., Wang, B., et al. (2010). Redox regulation of the stability of the SUMO protease SENP3 via interactions with CHIP and Hsp90. EMBO J. 29, 3773-3786. https://doi.org/10.1038/emboj.2010.245.

Yang, S.H., and Sharrocks, A.D. (2004). SUMO promotes HDAC-mediated transcriptional repression. Mol. Cell 13, 611-617.
Yeh, E.T. (2009). SUMOylation and De-SUMOylation: wrestling with life's processes. J. Biol. Chem. 284, 8223-8227. https://doi.org/10.1074/jbc.R800050200.

Yin, Y., Seifert, A., Chua, J.S., Maure, J.F., Golebiowski, F., and Hay, R.T. (2012). SUMO-targeted ubiquitin E3 ligase RNF4 is required for the response of human cells to DNA damage. Genes Dev. 26, 1196-1208. https:// doi.org/10.1101/gad.189274.112.

Yu, F., Shi, G., Cheng, S., Chen, J., Wu, S.Y., Wang, Z., Xia, N., Zhai, Y., Wang, Z., Peng, Y., et al. (2018). SUMO suppresses and MYC amplifies transcription globally by regulating CDK9 sumoylation. Cell Res. 28, 670-685. https://doi.org/10.1038/s41422-018-0023-9.

Yun, C., Wang, Y., Mukhopadhyay, D., Backlund, P., Kolli, N., Yergey, A., Wilkinson, K.D., and Dasso, M. (2008). Nucleolar protein B23/nucleophosmin regulates the vertebrate SUMO pathway through SENP3 and SENP5 proteases. J. Cell Biol. 183, 589-595. https://doi. org/10.1083/jcb.200807185.

Zhang, X.D., Goeres, J., Zhang, H., Yen, T.J., Porter, A.C., and Matunis, M.J. (2008). SUMO-2/3 modification and binding regulate the association of CENP-E with kinetochores and progression through mitosis. Mol. Cell 29, 729-741. https://doi.org/10.1016/j. molcel.2008.01.013.

Zhao, Y., Wang, Z., Zhang, J., and Zhou, H. (2016). Identification of SENP1 inhibitors through in silico screening and rational drug design. Eur. J. Med. Chem. 122, 178-184.

Zunino, R., Schauss, A., Rippstein, P., Andrade-Navarro, M., and McBride, H.M. (2007). The SUMO protease SENP5 is required to maintain mitochondrial morphology and function. J. Cell Sci. 120, 1178-1188. https://doi.org/10.1242/jcs.03418.

Zunino, R., Braschi, E., Xu, L., and McBride, H.M. (2009). Translocation of SenP5 from the nucleoli to the mitochondria modulates DRP1-dependent fission during mitosis. J. Biol. Chem. 284, 17783-17795. https://doi.org/10.1074/jbc.M901902200. 